\title{
The Use of Standardized DSM-5 Symptoms Increases the Likelihood of a False-Positive Diagnosis of Bipolar Disorder in Children Aged 6-12 in the United States
}

\author{
Chaandini Pandeti ${ }^{1}$ and Mary C. Boyes ${ }^{1}$ \\ 'Virginia Commonwealth University, Richmond, VA, USA \\ DOI: https://doi.org/10.47611/jsr.v10i1.1037
}

\begin{abstract}
$\underline{\text { ABSTRACT }}$
Over the past ten years, there has been a $10 \%$ increase in the misdiagnosis of psychiatric disorders in the United States. The increase is notably important as the rate of misdiagnosis of pediatric bipolar disorder is on the rise. I studied causes of misdiagnosing bipolar disorder, specifically in children, and analyzed why psychiatrists continue to misdiagnose children despite knowing that children are still growing and have radical symptoms, in order to develop a strategy that decreases the number of misdiagnoses of pediatric bipolar disorder. Several studies have shown that one of the leading causes of misdiagnosis includes the broad symptoms listed in the Diagnostic and Statistical Manual of Mental Disorders, Fifth Edition (DSM-5). The revisions to the DSM-4 involved the addition of 15 disorders. The symptoms listed in the DSM-5 are targeted towards adults, however, are being used to diagnose children. I analyzed longitudinal studies using national data to assess what percent of children are misdiagnosed with the disorder. The study showed that about $2.5 \%$ of the children were diagnosed with bipolar disorder based on the symptoms of the DSM-5. However, at the end of the study, the children no longer presented the bipolar disorder symptoms they were diagnosed by. The results from the study supported the claim that children are being over-diagnosed with bipolar disorder. I hypothesize that children should not be diagnosed with severe disorders until they are older and fully developed, unless they have severe symptoms that impair daily functioning. Based on the results of this study, pediatric bipolar disorder should be diagnosed by alternative diagnostic methods, such as using collateral history and therapy as opposed to prescribing strong medications. It is crucial that the misdiagnosis rate is addressed because children should not be dependent on such medications as their age is the crucial point for development.
\end{abstract}

\section{Introduction}

The Diagnostic and Statistical Manual of Mental Disorders, Fifth Edition (DSM-5) was released in 2013 with the addition of 15 new disorders. Along with the addition of new disorders, symptoms were either added to current disorders to include any form of previous symptoms to fall under the given disorder or symptoms were merged with others that fell under the given disorder. According to "The New Crisis of Confidence in Psychiatric Diagnosis," Allen Frances (2013), former chairman of the DSM-4's Task Force, claimed that the changes in DSM-5 may lead to highfalse positive rates and unnecessary treatments due to the misdiagnosis (p. 1). Based on recent statistical data, including the $10 \%$ increased misdiagnosis rate, it is evident that the DSM- 5 contributed to this increased rate for many reasons. The DSM-5 altered the requirement for number of symptoms that are needed in order to diagnose oneself with a disorder. For some behavioral disorders, including bipolar disorder, the number of symptoms that had to be present in an individual decreased. Due to the vague requirements of disorders, it was easier for a person to diagnose themselves with a severe disorder based on one or two symptoms. Additionally, people with simple stress symptoms were able to diagnose themselves with a mental disorder. Frances provided an example of a person who met the criteria of Somatic Symptom Disorder simply because he was concerned about his health. Because of the vague symptoms 
listed in the manual, people could easily diagnose themselves with behavioral or mental disorders based on daily stressors, which could contribute to the increased false-positive cases. There has been an increase in the misdiagnosis rate from the past ten years and the current misdiagnosis rate for children is about $43 \%$ in the United States. Symptoms listed in the DSM-5 are targeted towards adults, however, these symptoms are also being used to diagnose children. This can be harmful to children because it may lead to an increase in false-positive diagnoses since symptoms in children may be a part of developmental stages since children are still maturing. Bipolar disorder is one of the common disorders that children are misdiagnosed with because of their hypomania, which is a major spectrum of bipolar disorder. Hypomania is described as elevated activity or energy, which may be misinterpreted in children because children are typically hyperactive and possess many hypomanic symptoms.

However, these symptoms could be attributed to the simple fact that children have not yet fully matured as they are growing and their prefrontal cortex, which plays a major role in logical reasoning and controlling emotions, has not yet fully developed. Misdiagnosing children with bipolar disorder is especially harmful because treatment for bipolar disorder includes administration of mood stabilizers. Children who have been given a false-positive diagnosis would unnecessarily have to take mood stabilizers which can cause a permanent change in their brain chemistry. The altered brain chemistry can cause lifelong consequences because children will be dependent on mood stabilizers and their brain would have to work to adapt with the new doses of drugs. If children who were falsely diagnosed with bipolar disorder are given medications that provide a supplement of serotonin or gabapentin, which are neurotransmitters involved in bipolar disorder, they may become extremely dependent on those medications which can lead to behavioral disorders in the future if they stop taking the medication, such as depression. This is because the brain adapts to the new threshold of chemicals and requires an increased dose in order to produce the same effect in an individual. The medications in children would incidentally cause children to become dependent on them regardless of if they had the behavioral disorder or not. Since the DSM-5 had a 10\% increase in the number of total disorders in the manual and broader symptoms associated with disorders, there has been an increase in the misdiagnoses rate which may have been due to broad symptoms that may apply to anyone causing people with simple stress symptoms to be categorized as having a severe mental disorder. Children are more susceptible to being misdiagnosed with a behavioral disorder due to their extreme behaviors and psychiatrists may fail to realize that the mere explanation for their behavior could be that children are still growing and do not have a fully developed brain.

\section{Vague Symptoms in DSM-5 and Easy to Misdiagnose}

The DSM-5 had many revisions made from the DSM-4, including the addition of 15 new disorders and new criterion for many disorders, such as hyperactivity disorder. The criterion in the DSM-5 for several behavioral disorders, including bipolar disorder, are too broad for psychiatrists or clinicians to solely rely on this manual to diagnose a patient and additionally, the boundaries of the symptoms are unclear. About $25 \%$ of the USA population are able to diagnose themselves with a psychiatric disorder, which is alarming because diagnosing oneself with a disorder should not be that simple.

In "Psychiatric Epidemiology and the False Positives Problem," Jerome C. Wakefield (2015) stated that the DSM-5 was a missed opportunity to address the misdiagnoses problems from the DSM-4 and that the expansiveness of the DSM-5 has led people with normal, average stress symptoms to believe that they have a psychiatric disorder due to how vague and general the symptoms of new disorders are. Wakefield also stated that false positives most often occur when harmful symptoms are mislabeled without explicitly stating the definition of the disorder dysfunction requirement (p. 2). Many symptoms in the DSM-5 are too vague and do not have specific boundaries in that individuals do not know what symptoms would fall under the disorder. Due to these unclear boundaries of symptoms, individuals may think that their simple stress symptom falls under the list of major symptoms of a disorder and start to worry about a potential diagnosis. In accordance with Wakefield's argument of the vagueness of DSM-5 symptoms, in "Bipolar Disorder Diagnosis: Challenges and Future Directions," Phillips and Kupfer (2013) stated that there are no clear boundaries between diagnosing bipolar 1 and bipolar 2 disorder in the DSM-5. DSM-5 has eight types of mood 
disorders, many of which share similar symptoms. Common symptoms for several disorders make diagnosing an individual difficult because there is no clear distinction on what separates a symptom in one disorder compared to the other. Bipolar disorder is one of the many disorders that are misdiagnosed frequently. Phillips and Kupfer argued that a major reason for the difficult diagnosis is the challenge of differentiating bipolar disorder type I or II from unipolar depression - an illness characterized by recurrent depressive episodes - especially in patients who present a depressive episode and in those with no clear history of mania or hypomania (p. 4). Phillips and Kupfer analyzed the symptoms of unipolar disorder and the two types of bipolar disorder and stated that unipolar depression is reportedly the most frequent misdiagnosis in patients with bipolar disorder, especially in bipolar disorder type II, because patients with this illness, by definition, never experience an episode of mania (p. 5). These misdiagnosis mishaps show how various behavioral disorders have unclear boundaries to differentiate between. The vagueness of the symptoms makes it difficult to distinguish whether or not a disorder is actually present in an individual. If a psychiatrist relies heavily on using the DSM-5 to diagnose patients, the diagnosis could have a high possibility of being a false-positive diagnosis based on how unclear the symptom requirements are.

In "Major Depressive Episode: Is it Bipolar 1 or Unipolar Disorder?" John J. Miller (2018) agreed with Phillips and Kupfer's argument that bipolar disorder is misdiagnosed with unipolar disorder. Miller analyzed a survey study conducted in 1996 on the National Depressive and Manic-Depressive Association chapters to distribute to groups who have been diagnosed with bipolar disorder. The results of this study showed that out of the 600 patients who were diagnosed with bipolar disorder, $69 \%$ of the patients were misdiagnosed, with the most common misdiagnosis to be unipolar disorder. Miller claimed that the DSM-5 criteria for a major depressive episode is identical for both a unipolar depression and a bipolar depression, which can cause a confusion in differentiating which disorder is accurate for the individual presenting the symptom. Similar studies conducted on children for a follow-up study of childhood depression showed how unipolar disorder and bipolar disorder are often times misdiagnosed because patients typically do not get checked for having hypomanic symptoms. A possible reason for the lack of surveillance of hypomania is because mania is not considered as life-threatening as depression would be. Depressive symptoms are more prevalent, and patients may feel that depressive symptoms are more noticeable since those symptoms impair their daily functions.

In "Diagnostic Issues in Bipolar Disorder," Jules Angst et al. (2003) agreed that the diagnostic criteria for diagnosing bipolar disorder hypomania is too broad and claimed that there is increasing evidence for the existence of a broad spectrum of bipolar disorders. Angst et al. stated that the use of a vaguer definition of hypomania increased the prevalence rates of bipolar 2 disorders and minor bipolar disorders. Angst et al. argued that hypomanic symptoms were more difficult to diagnose since patients rarely go to see a psychiatrist or clinician to get treatment for hypomania. Angst et al. stated that hypomanic symptoms are often not perceived subjectively or reported spontaneously because they are socially acceptable, if not advantageous, and cause no human suffering (p. 2). Due to this social norm and acceptance of hypomanic symptoms, Angst et al. claimed that patients are often times misdiagnosed with major depression since they go to get treatment for the more depressive symptoms because these symptoms are often times more harmful and socially unacceptable. The broad definitions for hypomania and social acceptance of such symptoms contribute to the misdiagnosis rate and make is difficult to distinguish what behavioral disorder the symptoms may be attributed to.

\section{Many Children are Misdiagnosed with Bipolar Disorder}

Hypomania is defined as a period of abnormally elevated mood and abnormally increased goal-directed activity and energy. Since children typically have temper tantrums and are extremely active, children are often misdiagnosed as having bipolar disorder, or even ADHD, as ADHD is frequently misdiagnosed as bipolar disorder. Psychiatrists may be subject to having confirmation bias or implicit bias while diagnosing children which contributes to the misdiagnosis crisis. Because children are still growing and their prefrontal cortex has not yet fully developed, their age is more susceptible to be misdiagnosed with bipolar disorder based on their hypomanic symptoms. Symptoms in the DSM-5 
may also cause confusion while diagnosing children because the symptoms listed are targeted towards diagnosing adults, not children. Symptoms presented in adults may look different than symptoms children have.

In "The Misidentification of Trauma Symptoms as Bipolar Disorder in Youth," Hillary Doyle Fitzsimmons (2015) claimed that the rapid increase in juvenile bipolar disorder diagnoses began in the mid-1990s headed by the research of Janet Wozniak, Joseph Biederman, Barbra Geller, and her colleagues. Fitzsimmons claimed that bipolar disorder in youth was more common than once thought and began to identify common symptoms among youth. Fitzsimmons analyzed a recent study by Kathleen Merikangas and colleagues of the National Institute of Mental Health (2011) and analyzed national data of over 10,000 adolescents between the ages of 13 and 18 and found that 2.5\% of youth met criteria for bipolar disorder in their lifetime and 2.2\% met criteria within a given year. Merikangas and colleagues also found $1.7 \%$ of the sample to display mania alone within a given year (p. 17). Fitzsimmons claimed that these results showed how youth are largely being diagnosed with bipolar disorder within a given year. Fitzsimmons further studied the group data and analyzed that half of these youth no longer demonstrated symptoms of hypomania or mania in their later years, which indicates that these youths no longer met the criteria for bipolar disorder. Fitzsimmons claimed that $55 \%$ of the youth hadn't expressed symptoms of mania in their adulthood demonstrating that the manic symptoms that were diagnosed when they were younger did not actually progress into bipolar disorder. Fitzsimmons also claimed that symptoms such as hyperactivity or anger presented in childhood are not as stable or as well understood as those established in adulthood. Diagnoses given to children may not be accurate because the symptoms presented may not be permanent and could just be a part of their developmental stages. As Fitzsimmons' studies show, bipolar disorder symptoms were not prevalent in the children that were studied once they were reached their adulthood. This study demonstrates how behavioral symptoms in children may not be accurate or permanent. The symptoms may be due to their dynamic characteristics and developing bodies.

In accordance with Fitzsimmon's argument of children getting misdiagnosed based on child-like behaviors, Stuart L. Kaplan, a clinical professor of psychiatry at the Penn State College of Medicine, claimed in an article published by Newsweek (2011) that it is difficult to imagine the increased rate at which children are being diagnosed with bipolar disorder. Kaplan argued that there is evidence against the existence of pediatric bipolar disorder. Kaplan stated that a child diagnosed with bipolar disorder will have symptoms, such as mania or anger, that characterize the child's typical behavior, as Fitzsimmons stated in accordance. Kaplan also stated, "In this telling, an elementary-school-age child with the disorder may be chronically enraged and have several tantrums per day. But this only points to another problem with the diagnosis: it's nearly impossible to distinguish between children alleged to have bipolar disorder and those with straightforward anger-control issues," (p. 1). Kaplan and Fitzsimmons both argued that although children may express manic symptoms, it cannot be compared to the symptoms listed under hypomanic symptoms as per the DSM-5 because those symptoms are targeted towards adults. There are not enough guidelines to be able to tell what qualifies as a manic symptom in children or if the symptoms presented in children are simply part of their dynamic personalities that are developing.

In agreement with Fitzsimmons and Kaplan's argument regarding the difficulty in diagnosing bipolar disorder in children, Benjamin I. Goldstein (2012) noted in "Recent Progress in Understanding Pediatric Bipolar Disorder" that the Diagnostic and Statistical Manual of Mental Disorders (Fourth Edition, Text Revision) (DSM-IV-TR) symptom criteria for bipolar disorder are the same for children, adolescents, and adults. Goldstein claimed that pediatric bipolar disorder has been more prevalent in recent years leading to controversy in the validity of diagnosing bipolar disorder. Goldstein stated that there are developmental differences in how symptoms are presented among the youth as opposed to how they are presented in adults. Goldstein argued that since symptoms of mania is one of the major symptoms of bipolar disorder, silly, rambunctious, and/or impulsive behavior often characterizes childhood and adolescence and it is important, particularly in diagnosing hypomania, to be able to distinguish normal childhood behavior from psychiatric symptoms. Goldstein also claimed that in order to be considered a pathological symptom, behavior must be inappropriate to context and associated with a change in functioning (p. 17). Given that mania is a major symptom of bipolar disorder as stated in the DSM-5, children may be misdiagnosed with either bipolar disorder or ADHD, or even both, because the symptoms of ADHD and bipolar disorder are similar. Mania is often times difficult 
to diagnose in children as there are no clear boundaries in being able to identify if the mania is due to a child actually experiencing a hypomanic episode or if the child is simply extremely happy and hyperactive due to their young, immature age. Diagnosing a child with a behavioral or mental disorder can be especially challenging because children aren't able to express themselves properly or if they are experiencing a symptom. Psychiatrists have to rely on how children behave and make a conclusion based on those behaviors and what the child's parents assume. This process of diagnosing a child contributes to the misdiagnosis rate because there is no coherent way to confirm whether or not the child is actually experiencing the certain symptom in question.

In "Bipolar Disorder Often Misdiagnosed in Children," Jim Rosack stated that studies have indicated that between 40 percent and 90 percent of 320 children with bipolar disorder also have ADHD. David Rabnier (2013), a research professor at Duke University, stated that a common criticism of the criteria of ADHD has been that the symptoms reflect how the disorder presents in school age children and fails to capture how it presents in older adolescents and adults. Because of this, Rabnier claimed that some have argued that different symptom sets should be developed for different age groups, however, the new diagnostic criteria in the DSM-5 retain the same symptoms as before. This brings the question of whether or not these children actually have both disorders and if they are validated in taking the specific medications for both disorders.

Misdiagnosis rates for behavioral disorders are especially prevalent in children because they are progressing through developmental stages where they are establishing personalities that fit in with how they see themselves. Gifted children are often times misdiagnosed frequently because of their different thought patterns or behavior. In Misdiagnosis and Dual Diagnoses of Gifted Children and Adults: ADHD, Bipolar, OCD, Aspergers, Depression, and Other Disorders, James T. Webb et al. (2016) claimed that gifted children are more prone to being misdiagnosed with bipolar disorder or ADHD due to their differing thought processes compared to average children. Webb et al. stated that "Upon examination, these professionals discovered that many of these patients had been seriously misdiagnosed that, in fact, they were gifted individuals who were in situations in which the people around them did not sufficiently understand or accept behaviors that are inherent to people who are intellectually or creatively gifted," (p. 1). Webb et al. argued that the diagnosis of these gifted children was not valid because psychiatrists did not take into consideration that children may have different thought processes and may not express certain emotions. Diagnosing children, gifted or not, with behavioral disorders can be difficult as their personalities are dynamic and have not fully developed.

Often times when diagnosing patients, psychiatrists are subject to confirmation bias, which is inevitable because according to "Why Evolution May Favor Irrationality," Sharon Begley (2010) stated that we succumb to confirmation bias because it helps us evaluate arguments that are intended to persuade other people (p. 1). Humans are bound to having some form of bias because it helps us reason with ourselves and give an explanation for unknown conditions. Additionally, in "The Effects of the Confirmation Bias on Diagnostic Decision Making," Meagan Carleton Parmley (2006) stated that psychiatrists may form impressions based on the verbal context of their patient's comments, facial expression, or nonverbal behaviors (p. 64). Parmley analyzed various studies including diagnosing patients based on DSM-5 symptoms and claimed that these predisposed assumptions may lead psychiatrists to form inaccurate and premature impressions which can be problematic because once an initial diagnostic impression is formed, it is difficult to alter this impression (p. 65). Parmley studied how psychiatrists make assumptions on what the patient has based on initial symptoms and behaviors. Based on these findings, Parmley stated that having such predisposed biases can have adverse effects as it is more likely to cause a misdiagnosis. Initial assumptions are what cause misdiagnoses and can lead to harmful consequences, especially in children. Having confirmation bias and looking out for symptoms in children that match the symptoms list of the disorder the psychiatrist primarily had in mind can be detrimental to children. 


\section{Misdiagnosing Children with Bipolar Disorder is Harmful}

One of the treatments for bipolar disorder is prescribing mood stabilizers that aid in minimizing the high and low symptoms, including depression and manic episodes. Lithium drugs, which are commonly used to treat mood disorders, affect the structure of the brain as they are able to turn certain genes on or off through phosphorylation. These changes in gene expression effect the body's overall metabolism and receptor sensitivity. If children are given a falsepositive diagnosis for bipolar disorder, it can be harmful because children are then prescribed mood stabilizers which unnecessarily alter their brain chemistry.

In "Is Bipolar Disorder Being Over-Diagnosed?" James Myhre and Dennis Sifris (2018) stated that the broad interpretation of the APA guidelines for bipolar disorder contributed to the high rates of false-positive diagnosis of bipolar disorder and consequently results in children being exposed to bipolar therapy and medications. Timothy J. Legg in "What are the Long-Term Effects of Bipolar Disorder on the Body?" explained what the long-term effects are of such medications. Legg stated that exposing children to unnecessary treatment can cause numerous permanent side-effects including tremors, increased glucose and lipid levels in the blood, and eye pain or vision changes. The most common drugs used to treat bipolar disorder include Lithium, Zyprexa, Seroquel, Risperdal, Abilify, Geodon, and Clozaril. These drugs alter the levels of serotonin, noradrenaline, and dopamine that get released in the brain. In "Review of Lithium on Brain and Blood," Wise Young (2009) stated that lithium acts through multiple pathways to inhibit glycogen synthetase kinase-3beta (GSK3 beta). Young stated that GSK3 beta phosphorylates and inhibits nuclear factors that normally turn on cell growth and protection programs, including the nuclear factor of activated $\mathrm{T}$ cells (NFAT) and WNT/beta-catenin, which is responsible in controlling cellular functions. Young also stated that lithium is able to turn certain genes on by inhabiting a number of kinases and phosphates which in turn affect many systems including metabolism, receptor sensitivity, and inflammation. These alterations may be detrimental for children as their bodies aren't fully developed yet. Lithium medications are especially harmful if children were given a false-positive and were prescribed unnecessary and powerful medications because children will now have to depend on these medications just to reach the threshold their brain had before taking the medications.

The National Institute of Mental Health (2016) stated that possible side effects of taking lithium to treat mania or stabilize bipolar disorder include tremor in hands, irregular pounding of the heart, loss of coordination, and hallucinations. In "Children and Teens with Bipolar Disorder," Smitha Bhandari (2018) stated that most drugs for bipolar disorder were originally tested in adults, meaning that these drugs prescribed for bipolar disorder may not be suitable for children. Not doing proper testing on children with the medications that are used to treat bipolar disorder can be extremely detrimental as the effects would be unknown on children. Prescribing medication that essentially has not been confirmed to work in children can be threating to children's lives because they are unnecessarily being exposed to such strong medications. Bandari also stated that children are more prone to side effects of mood stabilizer drugs such as changes in blood sugar and cholesterol, which can cause permanent changes in the body functions, due to fact that their bodies are still developing and haven't yet built a strong immune system to aid in absorbing the strong medications. Misdiagnosing children with bipolar disorder can be detrimental because unnecessary medications can permanently alter a children's brain causing them to depend on such drugs throughout their life.

\section{Alternative Methods to Decrease the Misdiagnosis Rate of Bipolar Disorder in Children}

Because the DSM-5 is the handbook used by many health care professionals around the world, many psychiatrists resort to using the DSM-5 when diagnosing a patient. However, this diagnosis process has led to an increase in misdiagnosis rate as many stress symptoms are characterized as a major disorder. Rather than using the DSM-5 to diagnose a patient and instantly coming to a conclusion on the type of mental disorder a patient has, psychiatrists can use alternative methods, such as looking at family history, questionnaires, or therapy in order to help reduce the number of false-positive diagnoses. This is essential in diagnosing children because it can help reduce the misdiagnosis rate for children and preventing them from being dependent on severe medications. 
In "The DSM-5 Definition of Mental Disorder: Critique and Alternatives," Bruce A Thyer (2014) restated the DSM-5 definition of a disorder. The DSM-5 states that, "A mental disorder is a syndrome characterized by clinically significant disturbance in an individual's cognition, emotion regulation, or behavior that reflects a dysfunction in the psychological, biological, or developmental processes underlying mental functioning..." (p.3). Thyer stated that there are fundamental errors in reasoning when diagnosing a patient with a disorder based on the DSM-5. Thyer claimed that there are two logical errors in reasoning; one of the reasons is the mistake of circular reasoning which occurs in mental health when behavior is used to explain itself. This process of using circular reasoning can lead to diagnostic errors because psychiatrists focus primarily on the symptoms presented rather than the potential environment the children grew up in or rather their family history. Thyer argued that the definition in DSM-5 itself raises questions whether or not diagnosing someone with the symptoms listen is valid because the definition has reasoning flaws. Using behavior or trying to validate a certain symptom solely based on the DSM-5 stating that it is considered a disorder is a flaw in the diagnosing method because having a predisposed mindset that a patient has a symptom is what contributes to a false-positive diagnosis. A patient will believe that he or she has the disorder and try reasoning it out within themselves by searching for potential behaviors that may slightly fit the criterion, which shows that confirmation bias is inevitable.

Thyer claimed that there are viable alternatives to use when diagnosing clients rather than relying on the DSM-5. Thyer proposed symptomatic treatment as one alternative. Symptomatic treatment is when a psychiatrist views their patient as not necessarily having a disorder and is a form of cognitive-behavioral therapy where the psychiatrist would start with a blank slate and simply listen to the patient's concerns, understand why they are occurring, and either offer treatment or further define the symptoms that are causing distress. Thyer stated that psychiatrists could help the client going through distress by picking the aspect of the condition that seems most troubling. This method may help lessen confirmation bias because psychiatrists would not be allowed to diagnose a patient the day of the visit. Symptomatic treatment could also help lower the misdiagnosis rate because psychiatrists would be addressing the situational variables that could be causing the symptom rather than predominantly emphasizing dispositional attributes.

Thyer additionally stated that using the Functional Behavioral Assessment (FAB) is another alternative to use rather than the DSM-5. Thyer stated that a psychiatrist using FAB would develop operational descriptions of specific problem behaviors that the patient experiences. The psychiatrist would do so through clinical interviews and direct observations of the client's behavior preferably in the client's natural environment. Observing clients in their natural environment will help eliminate any confounding variables that may cause a false-positive diagnosis. Additionally, the psychiatrist would be able to observe the symptoms first-hand and not have to rely on assumptions based on what the client describes. Thyer claimed that FAB is an enviable alternative to using the DSM-5 because psychiatrists make hypothesis based on observations which help understand the function of the behavior. Thyer argued that observing the functions of the behavior would help understand how the person interacts with his or her environment, develop an etiological hypothesis, and adjust the intervention based on the results would strengthen the effectiveness of diagnosing patients.

Yet another way to prevent misdiagnosing patients, in particular children, based on the DSM-5 strategy, is to not diagnose children with a disorder until they are older, unless the disorder is life-threatening. In "To Diagnose or Not to Diagnose in Children's Mental Health? That is the Question," Robin Alter (n.d.) argued that parents cannot make a decision for their children based on questionnaires because they are not able to assess what exactly is going on in the child. Alter claimed that parents may have their own bias on their children and may use a disorder to justify a child's behavior. Children are simply too young to be diagnosed because they are still developing and many parts of their brain that control impulse and behaviors have not fully developed. There have not been enough studies done on children to diagnose them based on DSM-5 symptoms. Children are different in how they develop and what type of environment they grew up in. Because environment plays a substantial role in influencing child behavior, not diagnosing a child until they have fully developed or established a stable personality can be extremely helpful in reducing the misdiagnosis rate. Using the alternative techniques to help understand radical symptoms children present can help 
focus on the situational attributes and reduce bias in diagnosing children. In "Six Problems with Psychiatric Diagnosis for Children," Marilyn Wedge (2011) claimed that developmental delays are often medicalized and framed as psychiatric disorders in today's society. Wedge said, "Not all children grow and develop at the same rate. Some children learn to read at five, some at six, and some even later. But todays frantic parents seek out diagnoses and medication for a child who may simply be a late bloomer," (p. 1). Since psychiatrists are mostly taught to diagnose adults, it is critical to understand that children are different and should be diagnosed in alternative ways because they are mentally and physically different from adults.

\section{Conclusion}

The DSM-5 is a widely used handbook that health care professionals use to diagnose mental disorders. A patient may match their symptoms with a mental disorder and have the mindset that they do indeed have a mental disorder and get checked by a psychiatrist. This can lead to a confirmation bias while diagnosing a patient because of previous speculations of having a disorder. These assumptions based on the DSM- 5 contributed to an increase in the misdiagnoses rate because patients with simple stress symptoms were able to diagnose themselves with a mental disorder. The DSM5 has made it possible to have one symptom that falls under a major disorder to be considered mentally ill. The new disorders and broad symptoms that were added to the DSM-5 caused an increase in false-positive diagnoses because there are no clear boundaries within the symptoms. The DSM-5 is also used to diagnose children, which may be inaccurate because the DSM-5 is targeted towards adults and research has primarily been done on adults. Using the DSM-5 to diagnose children may result in a false-positive diagnosis because children could potentially have a symptom that is listed under a disorder, however, may not have been developed or could be a temporary symptom. Bipolar disorder is frequently misdiagnosed in children because of their hypomania. Misdiagnosing children with bipolar disorder can be harmful because the mood stabilizers that are used as a treatment for bipolar disorder can negatively affect a child's brain and cause children to be dependent on the drugs. Children are at a growing stage where their brains are still maturing and prescribing mood stabilizers to children will negatively impact the way a child's brain normally functions. There are many alternative methods to diagnose children rather than using the DSM-5, which was intended to be used to diagnose adults. Alternative methods that could be used to further research on misdiagnosis and how to prevent the increased rate include therapy, questionnaires, and family history. Using these methods will help decrease the misdiagnosis rate, especially for children, because it potentially eliminates bias while diagnosing patients. Rather than having a steady increase in the misdiagnosis rate, these methods can help bring the rate down and potentially have a better method to diagnosing patients. This will help in many ways because patients will be able to get an accurate diagnosis and not have to spend money on treatments or medications that are not necessary. Children will additionally not have to depend on high doses of medications and be able to grow and mature naturally.

\section{References}

Alter, R. (n.d.). To diagnose or not to diagnose in children's mental health? That is the question. Retrieved from http://www.docrobin.com/to-diagnose-or-not-to-diagnose-in-childrens-mental-health-that-is-the-question-2/

APA announces names of DSM-v work group members. (2008). Psychiatric Services, 59(6), 702-702. doi:10.1176/appi.ps.59.6.702

Angst, J., Gamma, A., Benazzi, F., Ajdacic, V., Eich, D., \& Rössler, W. (2003). Diagnostic issues in bipolar disorder. European Neuropsychopharmacology, 13, 43-50. doi:10.1016/s0924-977x(03)00077-4 
Bhandari, S. (2018, July 12). Children and teens with bipolar disorder. Retrieved from https://www.webmd.com/bipolar-disorder/guide/bipolar-children-teens\#2

Bipolar disorder. (2018, January 31). Retrieved from https://www.mayoclinic.org/diseases-conditions/bipolar-disorder/diagnosis-treatment/drc-20355961

Boseley, S. (2015, November 21). Generation meds: The US children who grow up on prescription drugs. Retrieved from https://www.theguardian.com/society/2015/nov/21/children-who-grow-up-on-prescription-drugs-us

Causes of bipolar disorder. (n.d.). Retrieved from https://www.webmd.com/bipolar-disorder/guide/bipolar-disordercauses\#1

Doyle Fitzsimmons, H., Foltz, Robert, \& Galezewski, James. (2015). The misidentification of trauma symptoms as bipolar disorder in youth. ProQuest Dissertations and Theses. 17-28. umi: 3645209

DSM-5: Frequently Asked Questions. (n.d.). Retrieved from https://www.psychiatry.org/psychiatrists/practice/dsm/feedback-and-questions/frequently-asked-questions

Epstein, H. (2015, November 17). Why it's so easy for doctors to misdiagnose kids. Retrieved from https://www.theatlantic.com/health/archive/2015/11/why-its-so-easy-for-doctors-to-misdiagnose-kids/416112/

Frances, A. (2013). The New Crisis in Confidence in Psychiatric Diagnosis. Annals of Internal Medicine, 159(3), 221-222. doi:10.7326/0003-4819-159-3-201308060-00655

Geller, B., Zimerman, B., Williams, M., Bolhofner, K., \& Craney, J. L. (2001). Bipolar disorder at prospective follow-up of adults who had prepubertal major depressive disorder. American Journal of Psychiatry, 158(1), 125-127. doi:10.1176/appi.ajp.158.1.125

Goldstein, B. I. (2012). Recent progress in understanding pediatric bipolar disorder. Archives of pediatrics \& adolescent medicine, 166(4), 362. doi:10.1001/archpediatrics.2011.832

Hirschfeld, R. M., Lewis, L., \& Vornik, L. A. (2003). Perceptions and impact of bipolar disorder. The Journal of Clinical Psychiatry, 64(2), 161-174. doi:10.4088/jcp.v64n0209

Kaplan, S. L. (2011, June 20). Mommy, am I really bipolar? Retrieved from https://www.newsweek.com/us-children-misdiagnosed-bipolar-disorder-67871

Legg, T. J. (2017, August 15). Bipolar disorder medications: Long-term effects on the body. Retrieved from https://www.healthline.com/health/bipolar-disorder/long-term-effects-on-the-body

Merten, E. C., Cwik, J. C., Margraf, J., \& Schneider, S. (2017). Overdiagnosis of mental disorders in children and adolescents (in developed countries). Child and Adolescent Psychiatry and Mental Health,11(1), 6-11.

doi:10.1186/s13034-016-0140-5

Miller, J. (2018, July 31). Major depressive episode: is it bipolar i or unipolar depression? Retrieved from: http://www.psychiatrictimes.com/special-reports/major-depressive-episode-it-bipolar-i-or-unipolar-depression. 
Myhre, J., \& Sifris, D. (2018, September 8). Is bipolar disorder being overdiagnosed? Retrieved from https://www.verywellmind.com/is-bipolar-disorder-being-overdiagnosed-4143329

Parmley, M. C. (2006). The Effects of the Confirmation Bias on Diagnostic Decision Making. The Effects of the Confirmation Bias on Diagnostic Decision Making, (1), 50-64. Retrieved from https://www.researchgate.net/publication/35905626 The effects of the confirmation bias on diagnostic decision making

Phillips, M. L., \& Kupfer, D. J. (2013). Bipolar disorder diagnosis: Challenges and future directions. The Lancet, 381(9878), 1663-1671. doi:10.1016/s0140-6736(13)60989-7

Richardson, E. (n.d.). Top 4 ways misdiagnosis drives up costs. Retrieved from https://www.healthcarefinancenews.com/news/top-4-ways-misdiagnosis-drives-costs

Stockman, J. (2012). Lifetime prevalence of mental disorders in U.S adolescents: Results from the national comorbidity survey replication-adolescent supplement (NCS-A). Yearbook of Pediatrics, 10, 385-387.

doi:10.1016/j.yped.2011.04.014

Thyer, B. A. (2015). The DSM-5 definition of mental disorder: critique and alternatives. Critical Thinking in Clinical Assessment and Diagnosis, 1,45-68. doi:10.1007/978-3-319-17774-8_3

Wakefield, J. C. (2015). DSM-5, psychiatric epidemiology and the false positives problem. Epidemiology and Psychiatric Sciences, 24(03), 188-196. doi:10.1017/s2045796015000116

Webb, James T., Amend, Edward R., Beljan, Paul. (2016). Misdiagnosis and dual diagnoses of gifted children and adults: ADHD, Bipolar, OCD, Aspergers, Depression, and other disorders. Retrieved from http://books.google.com

Wedge, M. (2011, May 23). Six problems with psychiatric diagnosis for children. Retrieved from https://www.psychologytoday.com/us/blog/suffer-the-children/201105/six-problems-psychiatric-diagnosis-children

Young, W. (2009). Review of lithium effects on brain and blood. Cell Transplantation, 18(9), 951-975. doi:10.3727/096368909x471251 